\title{
Failure analysis of an infinite unsaturated soil slope
}

\section{Annan Zhou PhD}

Senior Lecturer, School of Civil, Environmental and Chemical Engineering, Royal Melbourne Institute of Technology (RMIT), Melbourne, VIC, Australia Chun-Qing Li PhD

Professor, Head of School, School of Civil, Environmental and Chemical Engineering, Royal Melbourne Institute of Technology (RMIT), Melbourne, VIC, Australia (corresponding author: chunqing.li@rmit.edu.au)

\section{Jinsong Huang PhD}

Research Academic, Centre for Geotechnical and Materials Modelling, School of Engineering, the University of Newcastle, NSW, Australia

Increases in rainfall and groundwater level rising can cause more frequent failures of unsaturated soil slopes, resulting in large-scale landslides. The aim of this paper is to propose a methodology for prediction of the failure of an infinite soil slope subject to steady unsaturated flow conditions. Probabilistic models for soil porosity, friction angle, matric suction and saturation are developed. The probability of slope failure for different groundwater levels and infiltration (or evaporation) intensities is investigated. Numerical results show that, for a slope with deep groundwater, the slope failure is mainly controlled by the rainfall infiltration, whereas for shallow groundwater, the slope failure is governed by the location of the groundwater level. A merit of the proposed methodology is that the fluctuation of the soil porosity along the depth of a slope is modelled as a Gaussian random field. The paper concludes that the proposed methodology can be used for prediction of slope failures under steady unsaturated flow conditions. Accurate prediction of slope failures can prevent catastrophic consequences of landslides.

\section{Notation}

$c$

$f(y)$

G

$G_{\mathrm{S}}$

h

$h_{\mathrm{w}}$

$K_{\mathrm{r}}$

$K_{\mathrm{s}}$

$K_{\psi}$

$K_{0}$

$k$

$l$ cohesion at saturated state

void ratio

safety indicator for plane at specific location $y$

limit state function

specific gravity of soil

numerical integration element size/element size of random field

hydraulic head

relative hydraulic conductivity

soil's saturated hydraulic conductivity

unsaturated hydraulic conductivity

saturated hydraulic conductivity of soil at surface

fitting parameter for shear strength criterion

(Fredlund et al., 1996)

depth of phreatic surface

fitting parameter for Gardner's equation

total number of samples for Monte Carlo simulations soil porosity

trend function for soil porosity

porosity of soil at ground surface

probability of slope failure

infiltration intensity of rainfall in a steady state

degree of saturation

vector consisting of all the variables

depth of plane

parameter related to depth of weathering zone

thickness of soil layer

parameter in the van Genuchten (VG) model

\author{
parameter in VG model \\ bulk unit weight of soil \\ unit weight of water \\ scale of fluctuation \\ normalised infiltration intensity $\left(=q_{\mathrm{w}} / K_{0}\right)$ \\ slope angle \\ power reduction equation \\ mean value for variables involved in Monte Carlo \\ simulations \\ mean value for porosity profile \\ correlation function for porosity fluctuation \\ standard deviation for variables involved in Monte \\ Carlo simulations \\ standard deviation for porosity profile \\ vertical overburden stress \\ shear stress at the plane \\ shear strength at the plane \\ friction angle \\ friction angle of soil at ground surface \\ parameter in equation proposed by Gallipoli et al. \\ (2003) \\ $\omega_{\mathrm{n}} \quad$ pattern of fluctuation of soil porosity along the depth
}

\section{Introduction}

Rainfall infiltration and rise of groundwater level can cause the decrease of matric suction $(\psi)$ and increase of degree of saturation $(S)$, which can result in failures of unsaturated soil slopes. This is because the suction loss can severely weaken the 
shear strength of unsaturated soils and the increase of degree of saturation can change the shear stress distribution along the depth of a slope owing to the increase of the average bulk unit weight $(\gamma)$ of soil. Moreover, the shear strength of the soil is affected by the location of the phreatic surface and the rainfall intensity.

Various attempts have been made to estimate the safety of a slope subject to unsaturated flows, such as Iverson (2000); Cho and Lee (2002); Lu and Godt (2008); Ray et al. (2010); Travis et al. (2010); Li et al. (2013); Zhu et al. (2013) and so on. In general, most methods used for slope stability analyses with unsaturated steady seepages are deterministic, with the soil's mechanical and hydraulic properties treated as constants for a given soil layer. For example, Lu and Godt (2008) presented a framework for stability analysis of infinite slopes under steady unsaturated seepage conditions, considering a varying groundwater level and the variation of soil suction and moisture content above the groundwater level under steady infiltration conditions. It has been recently recognised that predicting the safety of slopes considering rainfall and groundwater level change inevitably involves uncertainties. For example, all the mechanical and hydraulic parameters of soils are determined by regression processes, based on experimental results, the variability of which cannot be ignored. Also, soil in the field cannot be ideally homogeneous along the depth, even if there is only one soil type. In other words, soil properties also exhibit spatial variability. For example, the porosity $(n)$ of the surface soil is usually greater than that of the undisturbed soil buried deeper than the groundwater level. Studies have shown that the porosity of weathered soil typically varies from 0.45 to 0.30 in the upper $10 \mathrm{~m}$ of the soil profile (Cornforth, 2005; Selby, 1993). As such, the friction angle of the deep soil is usually greater than that of the surface soil (Cornforth, 2005; Lu and Godt, 2008; Rowe, 1969). Experimental evidence (e.g. Cornforth, 2005; Rowe, 1969) shows that the friction angle for a given sandy soil can vary from $30^{\circ}$ to $55^{\circ}$ and is inversely linearly proportional to its porosity. Mitchell et al. (1972) also showed the inversely linearly proportional relationship between the friction angle and its porosity for a silty fine sand (a lunar soil simulant). Lu and Godt (2008) adopted such an inversely linearly proportional relationship between the friction angle and its porosity for their study on infinite slope safety. In their study, the friction angle for sandy silt, silt and loess varies from $33^{\circ}$ to $48^{\circ}$, owing to porosity change. In addition, according to previous investigation (Phoon and Kulhawy, 1999; Vanmarcke, 1977), soil properties do not change monotonically along the depth but can be fluctuant owing to the localised non-homogeneity.

Because of the uncertainty involved in predicting the slope safety, probabilistic methods for prediction of slope failure in the context of unsaturated conditions have been highlighted. The random finite-element method (RFEM) proposed by Griffiths and Fenton (1993) can generally be used to consider the uncertainty involved in all aspects of geotechnical engineering. It has been successfully applied to slope stability assessment, bearing capacity estimation and retaining wall design (Fenton and Griffiths, 2008). However, in the case of unsaturated conditions, RFEM can be very complicated because it involves implementation of hydro-mechanical coupled constitutive models for unsaturated soils, which requires complicated numerical techniques (Sheng, 2011) with inevitable low computational efficiency. It can also be very time-consuming, especially if the RFEM has to be combined with the Monte Carlo method to estimate the probability of slope failure.

Uncertainty can also be incorporated into analytical and semianalytical models to avoid the employment of finite-element analysis in predicting the probability of failure for slopes subject to unsaturated flows. For example, a number of probabilistic stability analyses (Cho, 2014; Santoso et al., 2011; Zhang et al., 2014) have been conducted for infinite slopes under rainfall infiltration considering the spatial variability of soil's saturated hydraulic conductivity $\left(K_{\mathrm{s}}\right)$. These analyses focus on the slope safety varying with the time under rainfall. However, few attempts have been made to study the joint effects of rainfall and varying groundwater level. In addition, in terms of soil properties, most probabilistic stability analyses only take into account the spatial variability of $K_{\mathrm{s}}$, but not soil porosity. Although $K_{\mathrm{s}}$ plays an important role in calculating profiles of suction and saturation in an unsaturated slope, soil porosity is more fundamental. This is because all key factors in unsaturated slope analysis, such as hydraulic conductivity $\left(K_{\mathrm{s}}\right)$, water retention behaviour $(S-\psi)$ and friction angle $(\phi)$, can be a function of soil porosity for a given soil type.

In this paper an attempt is made to propose a methodology for prediction of the reliability of an infinite soil slope subject to steady unsaturated flow conditions. In this methodology the fluctuation of the soil porosity $(n)$ along the depth $(y)$ is modelled as a Gaussian random field. The unsaturated Darcy's law, where the saturated hydraulic conductivity $\left(K_{\mathrm{s}}\right)$ is assumed to be a function of soil porosity (Carrier, 2003), is employed to calculate the suction distribution profile due to steady unsaturated flows related to rainfall and groundwater. A porositydependent water retention equation (Gallipoli et al., 2003; Zhou et al., 2012) is applied to calculate the saturation profile. Based on a comparative study in the paper by Sheng et al. (2011), the shear strength criterion proposed by Fredlund et al. (1996) is adopted to estimate the shear strength for unsaturated soils, in which the friction angle is also a function of soil porosity ( $\mathrm{Lu}$ and Godt, 2008). Finally, the Monte Carlo method is used to estimate the probability of slope failure and the most unsafe location for an infinite unsaturated slope.

\section{Formulation of slope failure}

Consider an infinite slope with angle $\theta$ (see Figure 1). According to Iverson (2000) and Travis et al. (2010), all forces within an infinite slope can be assumed to vary only in the 


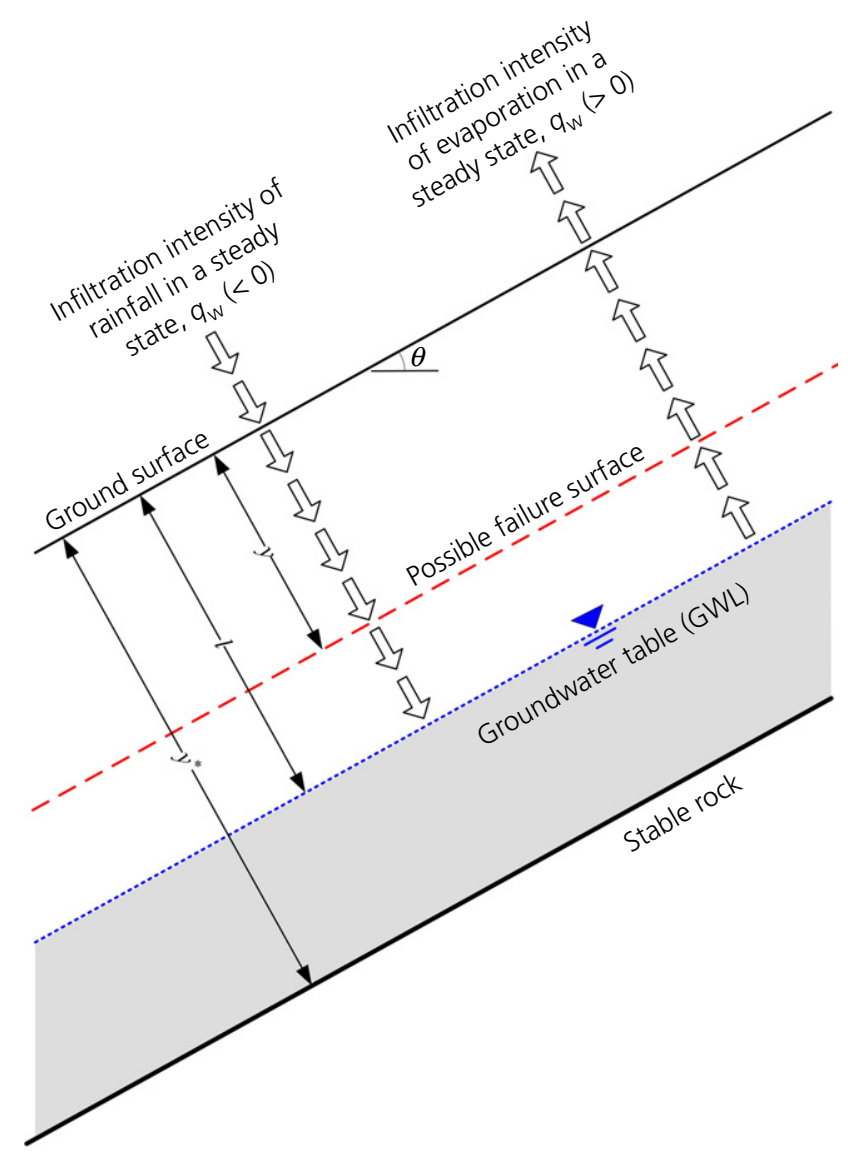

Figure 1. An infinite slope subject to steady unsaturated flows

direction perpendicular to the slope surface and the phreatic surface can be assumed to lie parallel to the slope surface at a known depth $(l)$. As shown in Figure $1, q_{\mathrm{w}}$ is the infiltration intensity of rainfall in a steady state, which is perpendicular to the slope surface. The value of $q_{\mathrm{w}}$ is set to be negative for steady wetting (rainfall infiltration) and positive for steady drying (evaporation).

For an infinite slope, as shown in Figure 1, the failure criterion for an arbitrary plane $(y)$ is that the shear stress at the plane exceeds the shear strength at the plane. In the risk analysis of slope failure, this criterion can be expressed in the form of a limit state function $(G)$ as follows

\section{1. $G(\boldsymbol{x}, y)=\bar{\tau}(\boldsymbol{x}, y)-\tau(\boldsymbol{x}, y) \quad\left(y \in\left[0, y^{*}\right]\right)$}

where $\bar{\tau}$ is the shear strength at the plane, $\tau$ is the shear stress at the plane, $y$ is depth of the plane and the thickness of soil layer is $y^{*}$. In this research, $y^{*}$ is set to be $10 \mathrm{~m}$ as a demonstration. $\boldsymbol{x}$ is the vector consisting of all the variables involved in calculating $\bar{\tau}$ and $\tau$. In the failure analysis, some or all of the variables $(\boldsymbol{x})$ can be assumed as random variables that follow some probability distributions, such as normal distribution. The variables in vector $\boldsymbol{x}$ and their values will be discussed later in Section 4.

The shear stress $(\tau)$ at an arbitrary plane is caused by the vertical overburden stress $\left(\sigma_{\mathrm{v}}\right)$ induced by the self-weight of soil mass and pore water. The shear stress for an arbitrary plane at depth $y$ can be written as

\section{2. $\tau(y)=\sigma_{\mathrm{v}}(y) \sin \theta \cos \theta$}

where the total vertical overburden stress $\left(\sigma_{\mathrm{v}}\right)$ can be calculated by integrating bulk unit weight $(\gamma)$ from the plane to the ground surface as follows

3. $\sigma_{\mathrm{v}}(y)=\int_{0}^{y} \gamma \mathrm{d} y=\gamma_{\mathrm{w}} \int_{0}^{y}\left[G_{\mathrm{s}}-G_{\mathrm{s}} n(y)+S(y) n(y)\right] \mathrm{d} y$

where $\gamma_{\mathrm{w}}$ is the unit weight of water $\left(=9.8 \mathrm{kN} / \mathrm{m}^{3}\right), G_{\mathrm{s}}$ is the specific gravity that is usually a constant for soils $(2.65 \sim 2.7$ for different soil types), $n$ is the soil porosity and $S$ is the degree of saturation. Both soil porosity $(n)$ and degree of saturation $(S)$ are functions of the depth $(y)$. Therefore, $n(y)$ and $S(y)$ are employed to represent the porosity profile and saturation profile along the depth, respectively. Owing to the high non-linearity of $n(y)$ and $S(y)$, Equation 3 usually cannot be integrated analytically. Here, the midpoint method is employed to conduct the numerical integration for Equation 3. The local truncation error for the midpoint method is in the order of $O\left(h^{3}\right)$, where $h$ is the numerical integration element size. In this paper, $h$ is set to be $10^{-3}$. The midpoint method is also known as the modified Euler method, the details of which can be found in Numerical Methods for Engineers by Griffiths and Smith (1991).

For the shear strength $(\bar{\tau})$ of unsaturated soils, a number of criteria have been proposed to describe the shear strength variation due to suction change as well as saturation change. Sheng et al. (2011) undertook a comparative study on eight widely used shear strength criteria for unsaturated soils and concluded that the simple criterion proposed by Fredlund et al. (1996) predicts unsaturated soil strength reasonably well. Therefore, the strength criterion proposed by Fredlund et al. (1996) is employed here to determine the shear strength for an arbitrary plane in the infinite slope

4. $\bar{\tau}(y)=\left\{\sigma_{\mathrm{v}}(y) \cos ^{2} \theta+[S(y)]^{k} \psi(y)\right\} \tan \phi(y)+c$

where $k$ is the fitting parameter for the shear strength criterion, $\psi$ is the matric suction, $\phi$ is the friction angle and $c$ is soil cohesion at the fully saturated state (assumed to be zero here). $\psi(y)$ and $\phi(y)$ are profiles of matric suction and friction angle along the depth, respectively. According to Sheng et al. (2011), 
parameter $k$ depends on the soil type, the value of which is usually greater than one. For example, for compacted kaolin clay (Thu et al., 2007), $k$ is calibrated to $1 \cdot 3$ and for Madrid Gray clay (Escario and Juca, 1989), $k$ is calibrated to $2 \cdot 8$.

Therefore, the limit state function of an arbitrary plane $(y)$ can be specified by combining Equations $2-4$ as follows

5.

$$
\begin{aligned}
G(\boldsymbol{x}, y)= & \gamma_{\mathrm{w}}\left(\cos ^{2} \theta \tan \phi(y)-\sin \theta \cos \theta\right) \\
& \left\{\int_{0}^{y}\left[G_{\mathrm{s}}-G_{\mathrm{s}} n(y)+S(y) n(y)\right] \mathrm{d} y\right\} \\
& +[S(y)]^{k} \psi(y) \tan \phi(y)
\end{aligned}
$$

From Equation 5, it can be seen that the limit state function for an arbitrary plane in the soil slope involves four depthdependent profiles, namely, the profile of soil porosity, $n(y)$, the profile of friction angle, $\phi(y)$, the profile of matric suction, $\psi(y)$, and the profile of degree of saturation, $S(y)$, all of which will be discussed in Section 3.

For a specific plane at the depth of $y\left(y \in\left[0, y^{*}\right]\right)$, the safety of this plane can be defined using the limit state function of Equation 1

6. $f(y)=\left\{\begin{array}{ll}0 & \text { if } G(\boldsymbol{x}, y) \geq 0 \\ 1 & \text { if } G(\boldsymbol{x}, y)<0\end{array}\right.$, for a given $y$

$f(y)$ is the safety indicator for the plane at a specific location y. $f(y)=1$ stands for the failure of plane $y$ and it is safe when $f(y)=0$. A failure of any plane in the slope leads to the failure of slope. The probability of slope failure of a plane at $y$ depth (i.e. $\left.P_{\mathrm{f}}(y)\right)$ can also be calculated by the Monte Carlo method

7. $\quad P_{\mathrm{f}}(y)=p[f(y)=1] \approx \frac{1}{N} \sum_{i=1}^{N} f_{i}(y)$

where $N$ is the total number of samples (i.e. the number of Monte Carlo simulations), $f_{i}(y)$ represents the value of $f$ (see Equation 6) at $y$ depth when the $i$ th sample of $\boldsymbol{x}$ is used for a simulation. $P_{\mathrm{f}}(y)$ is function of $y$ which varies between 0 and $y^{*}$. Therefore, the most unsafe location $(\tilde{y})$ can be defined as the plane with the maximum value of $P_{\mathrm{f}}(y)$ along the depth, that is, $\tilde{y}=\left.y\right|_{P_{\mathrm{f}}=\max }$.

\section{Models of soil properties}

\subsection{Profile of soil porosity, $n(y)$}

Soil that forms slopes is typically the result of chemical weathering, and mechanical transport and consolidation. In one aspect, the chemical weathering that results in the reduction of soil becomes more distinct with the decrease in depth because soil has more contact with destructive agents, such as the atmosphere, rainfall and temperature variation. In the other aspect, the consolidation pressure caused by soil self-weight is gradually decreasing with the decreasing depth. Consequently, for soils with the same chemical compositions, soil porosity at the ground surface is typically greater than that at deeper locations (Lu and Godt, 2008). Studies have shown that the porosity of weathered soil typically varies from 0.45 to 0.30 in the upper $10 \mathrm{~m}$ of the soil profile (Cornforth, 2005; Selby, 1993). Lu and Godt (2008) suggested a power reduction equation $(\lambda)$ to model the reduction of porosity along the depth

8. $n(y)=n_{0}-\lambda \Delta n, \quad \lambda=\left[1+\left(y_{\mathrm{w}} / y\right)\right]^{-1}$

where $n_{0}$ is the porosity of soil at the ground surface, $\lambda$ represents the power reduction equation, $\Delta n$ is the total change of soil porosity when $y$ changes from 0 to $\infty$, and $y_{\mathrm{w}}$ is a parameter related to the depth of the weathering zone. In the weathering zone, the porosity change of the soil along the depth is more prominent. The purpose of including a parameter for the weathering zone (i.e. $y_{\mathrm{w}}$ ) is to consider the nonlinear change of soil porosity along the depth. For a given total change of porosity (e.g. $\Delta n$ ), different $y_{\mathrm{w}}$ values lead to different patterns of soil porosity profile. Equation 8 can be applied to any slope analysis model if the non-linear decrease of soil porosity along the depth needs to be considered. Besides, it can be replaced by either a linear equation or more complex non-linear equations, depending on the borehole testing results and the precision requirement of the curve-fitting. Equation 8 is adopted in this research, but revised by considering the thickness of the soil layer to be $y^{*}$ rather than infinite, and then employed here as the trend function for soil porosity $(\bar{n})$. As shown in Figure 2(a), the soil porosity profile along the depth considering spatial variation can be expressed as

9. $\left\{\begin{array}{l}n(y)=\bar{n}(y)+\omega_{\mathrm{n}}(y) \\ \bar{n}(y)=n_{0}-\lambda \Delta n, \quad \lambda=\frac{1+\left(y_{\mathrm{w}} / y^{*}\right)}{1+\left(y_{\mathrm{w}} / y\right)}\end{array}\right.$

where $\Delta n$ is redefined as the total change of soil porosity between the ground surface and depth $y^{*}$ and $\omega_{\mathrm{n}}(y)$ is the pattern of fluctuation of the soil porosity along the depth, which can be modelled by a Gaussian random field through setting a mean $\left(\mu_{\mathrm{n}}=0\right)$, a standard deviation $\left(\sigma_{\mathrm{n}}\right)$ and a proper correlation function $\left(\rho_{i j}\right)$. The pattern of fluctuation, that is, $\omega_{\mathrm{n}}(y)$, is assumed to be unchanged for all samples of Monte Carlo simulation. In this research, the covariance matrix decomposition method (Cholesky decomposition) is employed to generate a random field (Fenton and Griffiths, 2008) and the correlation function, following Griffiths et al. (2011), is assumed as

10.

$$
\rho_{i j}=\exp \left(-2 \frac{\left|y_{i}-y_{j}\right|}{\delta_{\mathrm{y}}}\right)
$$



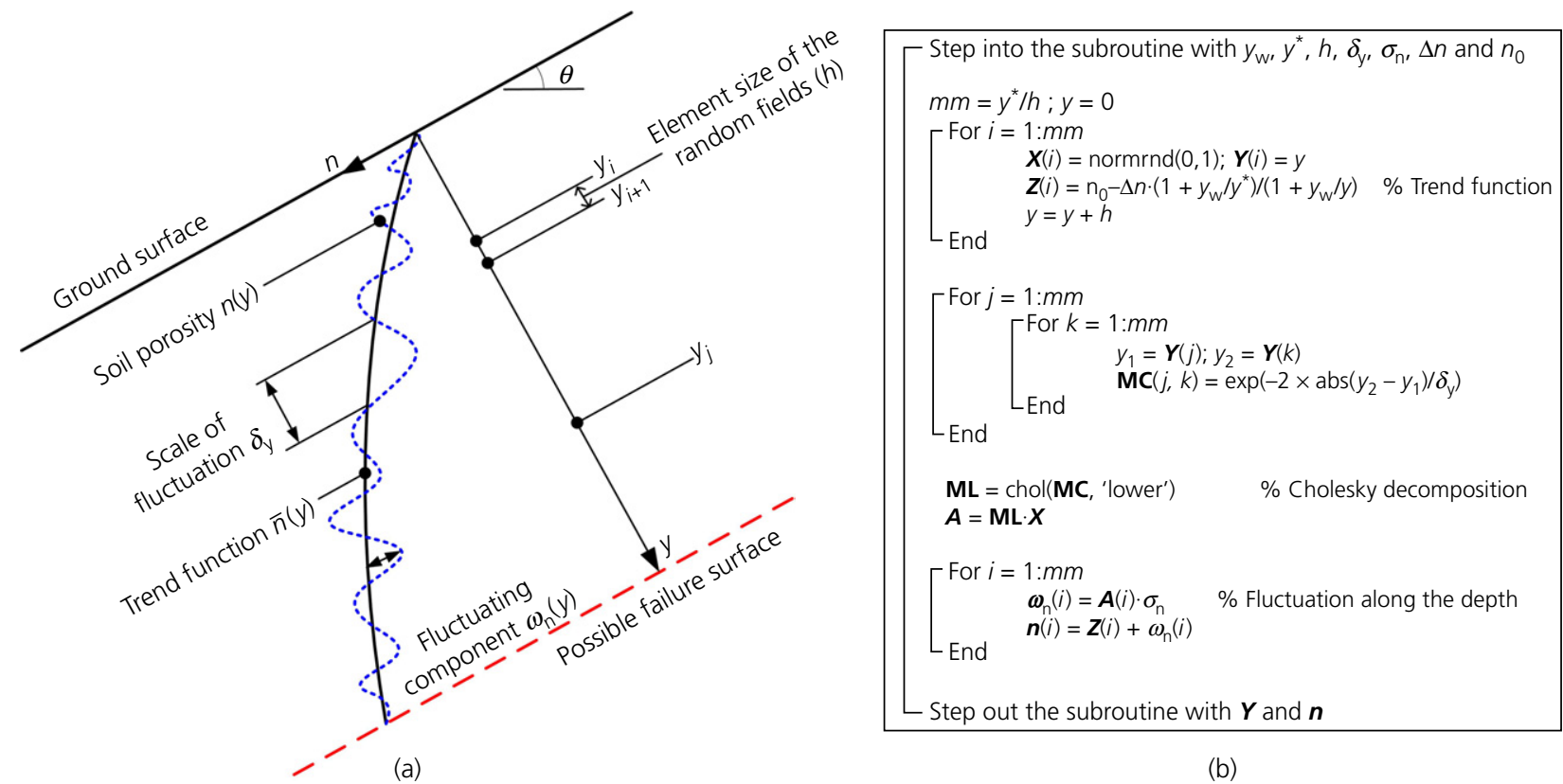

(b)

Figure 2. Modelling spatial variability of soil porosity through random fields: (a) schematic diagram; (b) pseudo-program

in which $y_{i}$ and $y_{j}$ are depths of two points within depth $y^{*}$ (i.e. $0 \leq y_{i} \leq y^{*}, 0 \leq y_{j} \leq y^{*}$ ), and $\delta_{\mathrm{y}}$ is the scale of fluctuation. Within the scale of fluctuation, values of porosity at two points show correlation, the tendency of which decreases with the distance between them. Porosities at any two points separated by distances beyond the scale of fluctuation have little correlation and behave independently. $h=y_{i+1}-y_{i}$ is the element size of the random field, which can be set to be identical to the numerical integration element size $\left(=10^{-3}\right)$. The pseudoprogram for modelling the profile of soil porosity by a Gaussian random field is also presented in Figure 2(b).

Figure 3(a) shows the calculated results of Equation 9 with different values of scale of fluctuation $\left(\delta_{\mathrm{y}}=1 \cdot 0,10\right.$ and $\left.100 \mathrm{~m}\right)$. As shown in Figure 3(a), with a constant $\sigma_{\mathrm{n}}$ value $(=0.006$ in this case), the predicted distribution of soil porosity fluctuates more if the scale of fluctuation decreases. The fluctuation pattern is also changed when the scale of fluctuation is different. When the scale of fluctuation is set to infinite, the predicted distribution curve is identical to the curve calculated by the trend function (see the dashed curve in Figure 3(a)). Figure 3(b) shows five different soil porosity profiles (i.e. profiles I, II, III, IV and V). Profile I shows constant porosity $\left(n_{0}=0 \cdot 6, \Delta n=0 \cdot 0\right)$ and profile II is the porosity profile represented by the trend function $\left(n_{0}=0 \cdot 6, \Delta n=0 \cdot 1, y_{\mathrm{w}}=2 \mathrm{~m}\right)$ with zero fluctuation $\left(\sigma_{\mathrm{n}}=0\right)$. Profiles III, IV and V show the simulation results of Equation 9 with different values of $\sigma_{\mathrm{n}}$ $(=0.006,0.018$ and 0.03$)$ at the same scale of fluctuation $\left(\delta_{\mathrm{y}}=1 \cdot 0 \mathrm{~m}\right)$ and trend function $\left(n_{0}=0 \cdot 6, \Delta n=0 \cdot 1, y_{\mathrm{w}}=2 \mathrm{~m}\right)$. Comparing the three different curves with different values of $\sigma_{\mathrm{n}}$ indicates that greater $\sigma_{\mathrm{n}}$ leads to a larger amplitude of fluctuation.

\subsection{Profile of friction angle, $\phi(y)$}

Experimental evidence (Cornforth, 2005; Rowe, 1969) shows that the friction angle $(\phi)$ for a given soil can vary from $30^{\circ}$ to $55^{\circ}$ and is inversely linearly proportional to its porosity $(n)$. $\mathrm{Lu}$ and Godt (2008) suggested the following linear relationship between the friction angle $(\phi)$ and porosity $(n)$, which will be adopted directly in this paper

11. $\phi(y)=\phi_{0}-\frac{\Delta \phi}{\Delta n}\left[n(y)-n_{0}\right]$

where $\phi_{0}$ is the friction angle of soil at the ground surface (i.e. $\left.n=n_{0}\right), \Delta \phi$ stands for the change of friction angle when the porosity change is $\Delta n$. The friction angle is independent of porosity if $\Delta \phi=0$.

\subsection{Profile of matric suction, $\psi(y)$}

According to Iverson (2000), the suction profile is the same as the distribution of the negative pore water pressure when $y \geq l$ (i.e. beneath the groundwater table)

$$
\text { 12. } \psi(y)=-\gamma_{\mathrm{w}}(y-l) \cos \theta \quad(y \geq l)
$$

When $y<l$, the suction profile can be calculated by way of the extended Darcy's law, which can be applied to the steady-state 


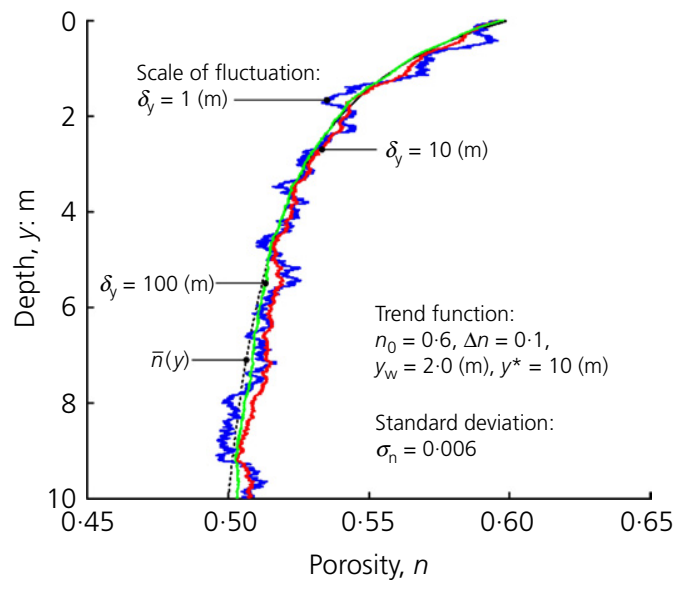

(a)
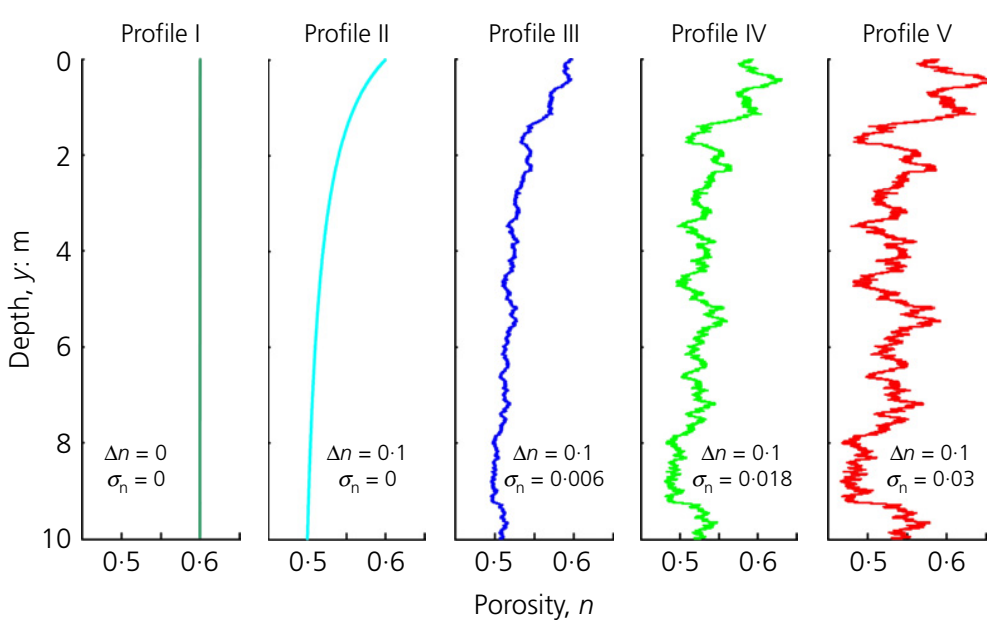

(b)

Figure 3. Soil porosity profiles simulated by way of random fields:

(a) simulated porosity profile with different scales of fluctuation;

(b) simulated porosity profile with different standard deviations

one-dimensional unsaturated conditions (Lu and Godt, 2008; Lu and Griffiths, 2004; Travis et al., 2010). The extended Darcy's law can be written as

$$
\text { 13. } K_{\psi} \frac{\mathrm{d} h_{\mathrm{w}}}{\mathrm{d} y}=q_{\mathrm{w}}
$$

where $h_{\mathrm{w}}$ is the hydraulic head. According to Iverson (2000), $h_{\mathrm{w}}=-\psi / \gamma_{\mathrm{w}}-y \cos \theta . q_{\mathrm{w}}$ is the infiltration intensity of rainfall that is perpendicular to the slope surface. The value of $q_{\mathrm{w}}$ is set to be negative for infiltration and positive for evaporation. $K_{\psi}$ is the unsaturated hydraulic conductivity at depth $y$. $K_{\psi}=K_{\mathrm{s}} K_{\mathrm{r}}$, in which $K_{\mathrm{s}}$ is the hydraulic conductivity for saturated soils and it is a function of soil porosity $(n)$

14. $K_{\mathrm{s}} \propto \frac{e^{3}}{1+e} \Leftrightarrow K_{\mathrm{s}}=K_{0} \frac{n^{3}}{(1-n)^{2}} \frac{\left(1-n_{0}\right)^{2}}{n_{0}^{3}}$

where $K_{0}$ is the hydraulic conductivity when soil porosity is equal to $n_{0} . K_{\mathrm{r}}$ is relative hydraulic conductivity, which can be a function of either degree of saturation $(S)$ or matric suction $(\psi)$. A number of equations have been proposed to specify the relative hydraulic conductivity function for unsaturated soils (Fredlund and Xing, 1994; Gardner, 1956; Mualem, 1976; van Genuchten, 1980). Following $\mathrm{Lu}$ and Griffiths (2004), the well-known relative hydraulic conductivity equation proposed by Gardner (1956) is employed here

15. $K_{\mathrm{r}}=\exp (-m \psi)$ where $m$ is a fitting parameter for Gardner's equation. Substituting Equations 14 and 15 into Equation 13 produces

16. $\left\{\begin{array}{l}\psi^{\prime}=\frac{\mathrm{d} \psi}{\mathrm{d} y}=-\frac{q_{\mathrm{w}} \gamma_{\mathrm{w}}}{K_{\psi}}-\gamma_{\mathrm{w}} \cos \theta \quad(y \leq l) \\ \psi=0(y=l)\end{array}\right.$

Equation 16 can be solved by using the finite-differential method, assuming $\psi^{\prime} \approx\left(\psi_{i+1}-\psi_{i}\right) / h . h\left(=y_{i+1}-y_{i}\right)$ is the grid size of the finite-difference interval, which can be set to be equal to the element size of the random field $\left(=10^{-3}\right)$. Equation 16 can be rewritten as

17. $\left\{\begin{array}{l}\psi_{i+1}=\psi_{i}-h \frac{\eta_{\mathrm{w}} \gamma_{\mathrm{w}}\left(1-n_{i}\right)^{2} n_{0}^{3}}{n_{i}^{3}\left(1-n_{0}\right)^{2} \exp \left(-m \psi_{i}\right)}-h \gamma_{\mathrm{w}} \cos \theta(y \leq l) \\ \psi_{i}=0, y_{i}=l, \text { when } i=1\end{array}\right.$

where $\eta_{\mathrm{w}}$ is the normalised infiltration intensity $\left(=q_{\mathrm{w}} / K_{0}\right)$. If $K_{\mathrm{s}}=K_{0}$ (i.e. porosity-independent), Equation 16 can be solved analytically as

18. $\psi(y)=-\frac{1}{m} \ln \left\{\left(1+\frac{\eta_{\mathrm{w}}}{\cos \theta}\right) \exp \left[m \gamma_{\mathrm{w}}(y-l) \cos \theta\right]-\frac{\eta_{\mathrm{w}}}{\cos \theta}\right\}$

Equation 18 can only be valid between the boundaries for evaporation and infiltration, that is

19. $\frac{\cos \theta}{\exp \left(m \gamma_{\mathrm{w}} l \cos \theta\right)-1}>\eta_{\mathrm{w}} \geq-\cos \theta$ 
Since $K_{\mathrm{s}} \leq K_{0}$, the valid value of $\eta_{\mathrm{w}}$ must be inside the evaporation/infiltration boundaries represented by Equation 19 . The analytic solution from Equation 18 can also be used to estimate the grid size adopted for Equation 17. As shown in Figure 4, the results obtained from Equation 17 match the analytic solution from Equation 18 very well when grid size is set to be $10^{-3}$.

Figure 5(a) shows the calculated suction profile for an infinite slope subject to different steady flow conditions $\left(\eta_{\mathrm{w}}=-0 \cdot 8,0\right.$,

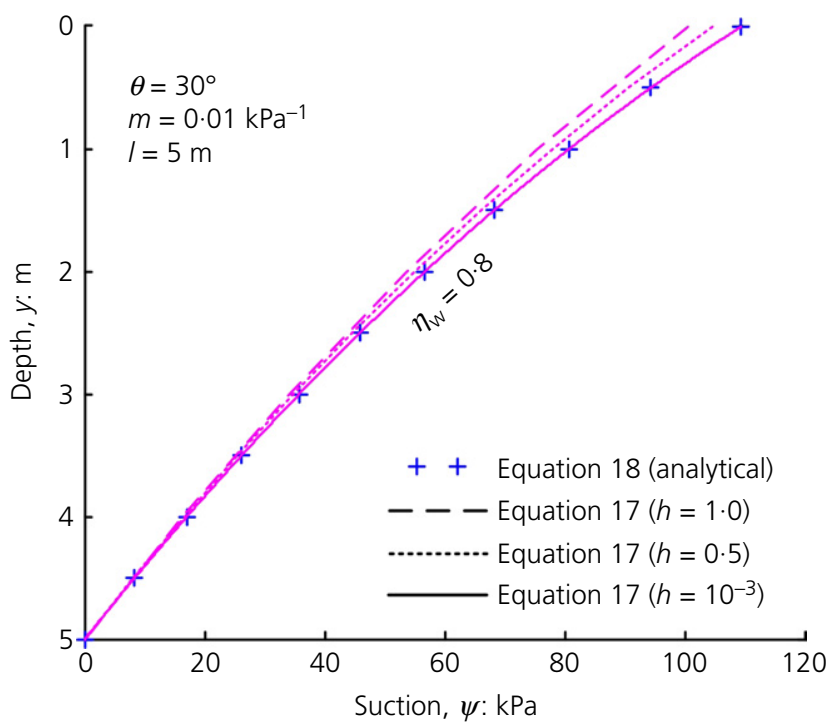

Figure 4. Determining the grid size for numerical integration

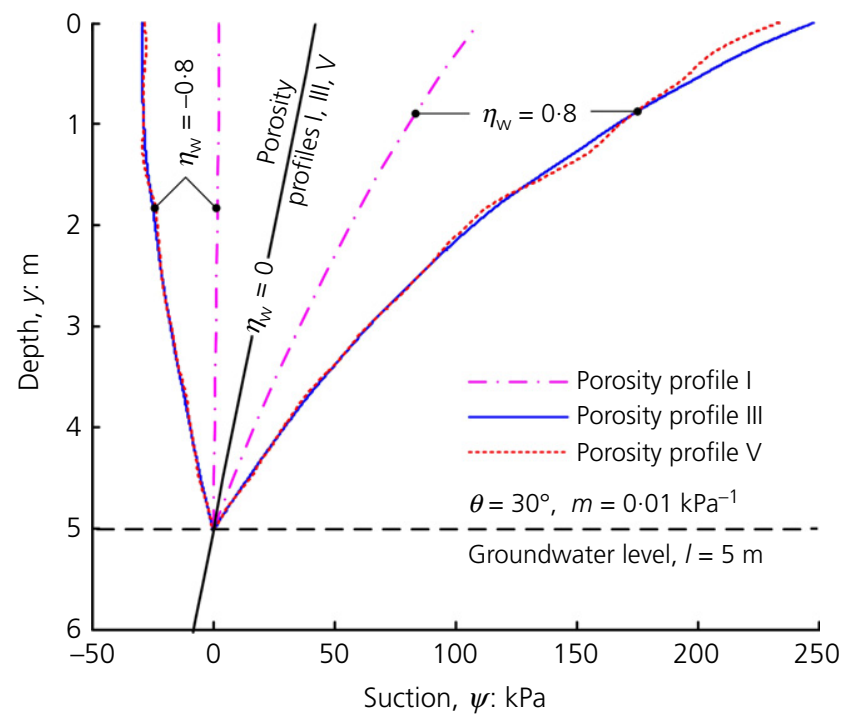

(a)

Figure 5. Simulated (a) suction profile and (b) saturation profile in the slope
$0 \cdot 8$ ) when the groundwater level is equal to $5 \mathrm{~m}$. Three different soil porosity profiles are involved (porosity profiles I, III and $\mathrm{V}$, as shown in Figure 3(b)). As shown in Figure 5(a), the suction distribution is independent of the porosity profile when $\eta_{\mathrm{w}}=0$. In other words, the suction profile is the same for soil porosity profiles I, III and IV when $\eta_{\mathrm{w}}=0$. When heavy evaporation occurs (e.g. $\eta_{\mathrm{w}}=0.8$ ) in the drought season, if $K_{\mathrm{s}}$ is a function of soil porosity that decreases along the depth in a fluctuating manner (see the calculated curves corresponding to porosity profiles III and V), the predicted suction is much greater than the suction calculated by assuming a constant soil porosity (see the calculated curve corresponding to porosity profile I). When heavy rain occurs (e.g. $\eta_{\mathrm{w}}=-0 \cdot 8$ ), if $K_{\mathrm{s}}$ is a function of soil porosity that decreases along the depth in a fluctuating manner (see the calculated curves corresponding to porosity profiles III and V), the predicted pore pressure (i.e. negative suction) is much greater than the pore pressure calculated by assuming soil porosity to be a constant (see the calculated curve corresponding to porosity profile I). Figure 5(a) also shows the suction distribution along the depth for soil profiles with different standard deviations. As shown in Figure $3(\mathrm{~b})$, the standard deviation $\left(\sigma_{\mathrm{n}}\right)$ of soil porosity is 0.006 and 0.03 for porosity profiles III and V, respectively. As shown in Figure 5(a), the fluctuation of the suction profile becomes more distinct with increasing amplitude of fluctuation of soil porosity.

\subsection{Profile of degree of saturation, $S(y)$}

For a given suction, the degree of saturation can be estimated by traditional water retention models, such as the $\mathrm{BC}$ model (Brooks and Corey, 1964), the VG model (van Genuchten,

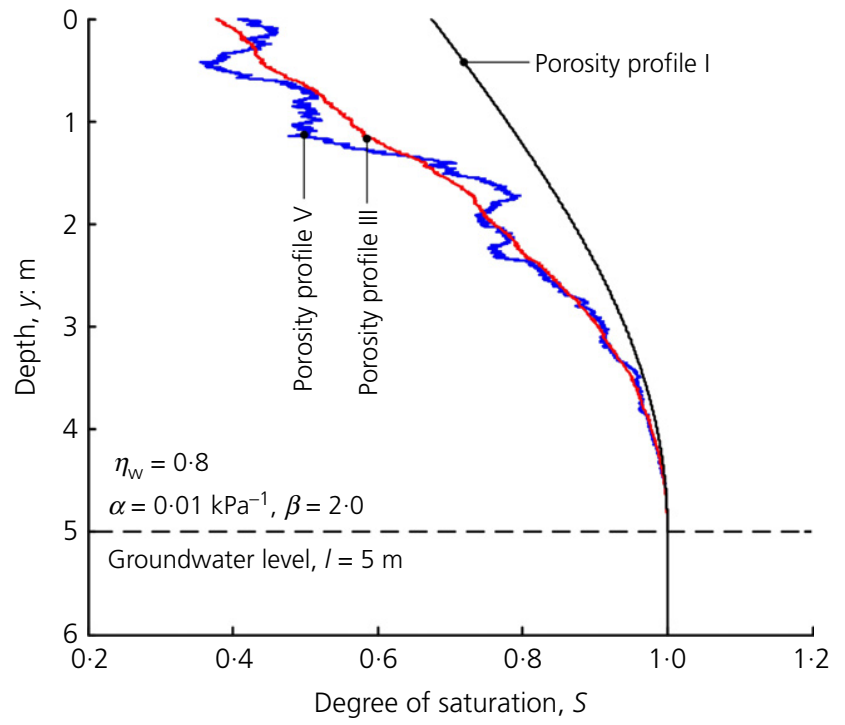

(b) 


$\begin{array}{cl}0.6 & 0.06 \\ 30 \cdot 0 & 3 \\ 0 \cdot 1 & 0.01 \\ 25 \cdot 0 & 2 \cdot 5 \\ 2 \cdot 0 & 0 \cdot 2 \\ 0.01 & 0.001 \\ 0.01 & 0.001 \\ 2 \cdot 0 & 0.2 \\ 1.5 & 0 \cdot 15 \\ 0 \sim 8 & 0.2 \\ -0.8 \sim 0.8 & 0.08\end{array}$

Total porosity change, $\Delta n$

Total friction angle change, $\Delta \phi$ : degrees

Weathered depth, $y_{w}: m$

Fitting parameter in Gardner's model, $m: \mathrm{kPa}^{-1}$

Fitting parameter in VG model, $\alpha: \mathrm{kPa}^{-1}$

Fitting parameter in VG model, $\beta$

Fitting parameter in Fredlund's model, $k$

$-0.8 \sim 0.8$

$0 \cdot 2$

Normalised infiltration intensity, $\eta_{\mathrm{w}}$

Table 1. Random variables in limit state function

1980) and the FX model (Fredlund and Xing, 1994). With respect to the traditional water retention models, degree of saturation is uniquely determined by the suction for a given soil and independent of all the other soil variables. However, recent research (Gallipoli et al., 2003; Sheng and Zhou, 2011; Sun et al., 2007; Tarantino, 2009; Zhou et al., 2012) showed that soil-water retention behaviour is also dependent on the porosity. Therefore, the traditional water retention equations can only be valid for a given soil with a specific porosity. A simple void-ratio-dependent water retention equation was proposed by Gallipoli et al. (2003) based on the VG model, which can be expressed as

20. $S=\left[\frac{1}{1+\left[\alpha\left(e / e_{0}\right)^{\varphi} \psi\right]^{\beta}}\right]^{1-(1 / \beta)}$

where $e$ is void ratio $(=n /(1-n)), \alpha$ and $\beta$ are two parameters in the VG model which are calibrated when the void ratio is set to $e_{0}$, and $\varphi$ is a parameter introduced to quantify the effect of the void ratio on the soil-water retention behaviour. If the value of $\varphi$ is set to zero, the term related to void ratio is removed and Equation 20 degenerates to the VG model. Zhou et al. (2012) suggested that $\varphi$ can be set to $1 /(\beta-1)$, based on the intrinsic constraints for a liquid-gas-solid mixture. Therefore, the profile of degree of saturation can be written as

21. $S(y)=\left\{1+\left[\frac{n(y)}{n_{0}} \frac{1-n_{0}}{1-n(y)}\right]^{\beta /(\beta-1)}[\alpha \psi(y)]^{\beta}\right\}^{(1 / \beta)-1}$

where $n(y)$ and $\psi(y)$ can be calculated from Equations 9 and 16, respectively. Figure 5(b) shows different saturation profiles obtained using Equation 21 according to different profiles for soil porosity (i.e. porosity profiles I, III and V, as shown in Figure 3(b)), when $l=5 \mathrm{~m}$ and $\eta_{\mathrm{w}}=0 \cdot 8$. As shown in Figure 5(b), for the evaporation condition, neglecting the porosity dependency will overestimate the degree of saturation in the soil. In addition, the fluctuation of soil porosity will result in a fluctuant profile of degree of saturation. Furthermore, a greater value of standard deviation of soil porosity will make the fluctuation of the saturation profile more distinct.

\section{Failure analysis of slope}

The probability of slope failure will be estimated by Monte Carlo method using Equation 1 as the limit state function. In Equation 1, some of the variables included in vector $\boldsymbol{x}$ are treated as basic random variables, as shown in Table 1 . These basic random variables are assumed to be of Gaussian distribution with known means and standard deviations. With the Monte Carlo method, a random sample is taken from the probability distribution function of each variable. Then, the sample is substituted into the limited state function $G$. If $G<0$ for a given depth $y$ in a slope, $f(y)=1$ (as shown in Equation 6). This process is repeated $N$ times, known as the sample size, from which $P_{\mathrm{f}}(y)$ can be determined by Equation 7. Meanwhile, the most unsafe location ( $\tilde{y})$ can be determined by finding the plane with a maximum probability of failure, namely the peak of the $P_{\mathrm{f}}-y$ curve. The fluctuation of soil porosity, which is simulated as a random field, will be included in the failure analysis. The fluctuation of soil porosity is controlled by values of $\sigma_{\mathrm{n}}$ and $\delta_{\mathrm{y}}$. In this section, $\delta_{\mathrm{y}}$ is set to be $1 \mathrm{~m} . \sigma_{\mathrm{n}}$ is set to be 0 and $0 \cdot 006$, which correspond to soil porosity profiles II and III (see Figure 3(b)), respectively. The fluctuation of soil porosity can be removed by setting $\sigma_{\mathrm{n}}=0$. In this section, $N$ is set to be 5000 to ensure the accuracy of simulations.

\subsection{Scenario 1: Deep groundwater level $(I=4.0 \mathrm{~m})$}

In the first scenario, the groundwater level is set to be $4.0 \mathrm{~m}$. The normalised infiltration ratio $\left(\eta_{\mathrm{w}}\right)$ is set to be from $-0 \cdot 8$ (rainfall) to $0 \cdot 8$ (evaporation). Figure 6 shows the probability of failure $P_{\mathrm{f}}(y)$ with respect to the location of the failure plane. As shown in Figure 6, for a slope with a deep groundwater 


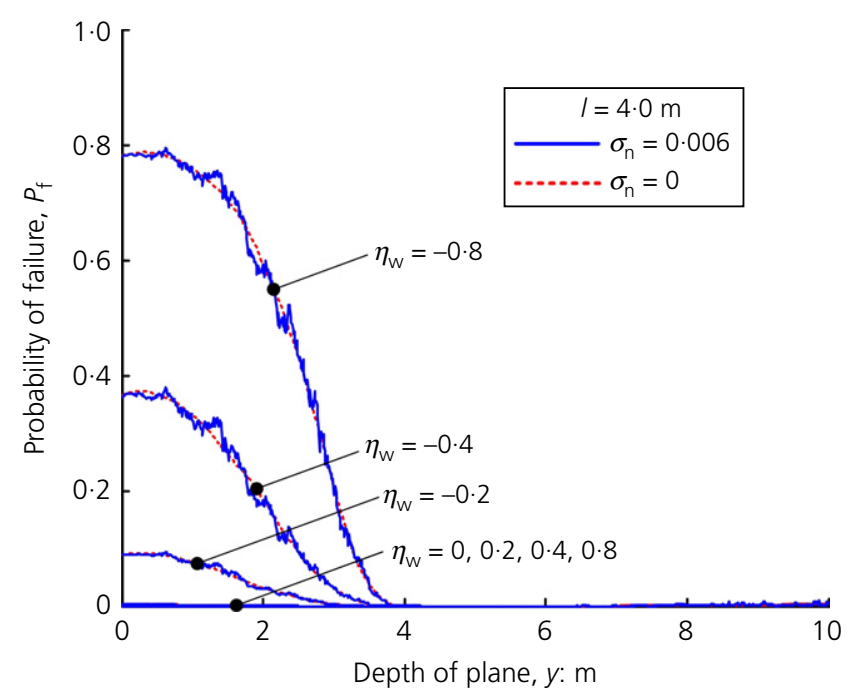

Figure 6. Probabilities of failure for a slope with a deep groundwater level

level, the slope failure is mainly controlled by the rainfall. For the equilibrium condition (i.e. $\eta_{\mathrm{w}}=0$ ), the probability of failure for the slope is almost equal to zero. The probability of failure of the slope markedly increases with the increase of the magnitude of the infiltration ratio for rainy conditions $\left(\eta_{\mathrm{w}}<0\right)$. The most unsafe locations for rainy conditions $\left(\eta_{\mathrm{w}}<0\right)$ are all less than $1 \mathrm{~m}$, which is much higher than the groundwater level. Therefore, the failure caused by rainfall occurs above the groundwater level. The fluctuation of soil porosity will cause the fluctuation of probability of failure along the depth. However, the difference of the probability of failure caused by the monotonic and fluctuating soil porosities is relatively small.

\subsection{Scenario 2: Medium groundwater level $(I=2.0 \mathrm{~m})$}

The groundwater level is set to be $2.0 \mathrm{~m}$ in the second scenario. Similarly to the first scenario, the normalised infiltration ratio $\left(\eta_{\mathrm{w}}\right)$ is set to be from $-0 \cdot 8$ (rainfall) to $0 \cdot 8$ (evaporation). The value of $\sigma_{\mathrm{m}}$ is set to be 0 and 0.006 for monotonic and fluctuating changes of soil porosity along the depth, respectively. Figure 7 shows the probability of failure $P_{\mathrm{f}}(y)$ with respect to the location of the failure plane. The probability of failure decreases from around 0.8 to 0.7 for a heavy rainfall $\left(\eta_{\mathrm{w}}=-0 \cdot 8\right)$ when the groundwater level rises from $4.0 \mathrm{~m}$ (the first scenario) to $2 \cdot 0 \mathrm{~m}$ (the second scenario). For the equilibrium and drier conditions $\left(\eta_{\mathrm{w}} \geq 0 \cdot 0\right)$, the probability of failure increases from around $0 \cdot 0$ to $0 \cdot 1$ due to the groundwater level rise. For a light rainfall $\left(\eta_{\mathrm{w}}=-0 \cdot 2\right)$, the probability of failure almost remains the same $(\sim 0 \cdot 1)$ for both scenarios. However, the most unsafe location is different for the two scenarios. In the first scenario, the most unsafe location is shallow and above the groundwater level for a light rainfall. For the second scenario, the most unsafe location is deep and beneath the

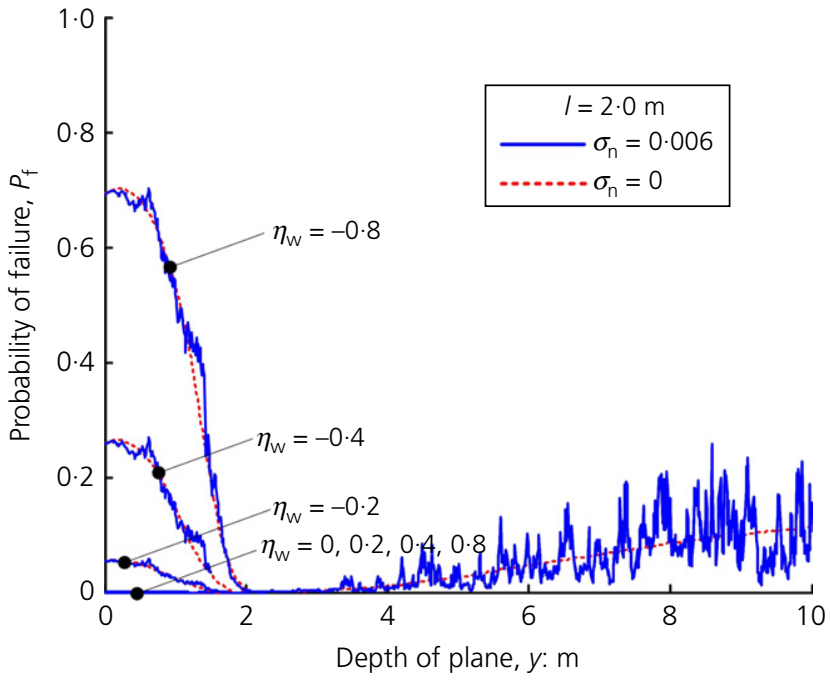

Figure 7. Probabilities of failure for a slope with a medium groundwater level

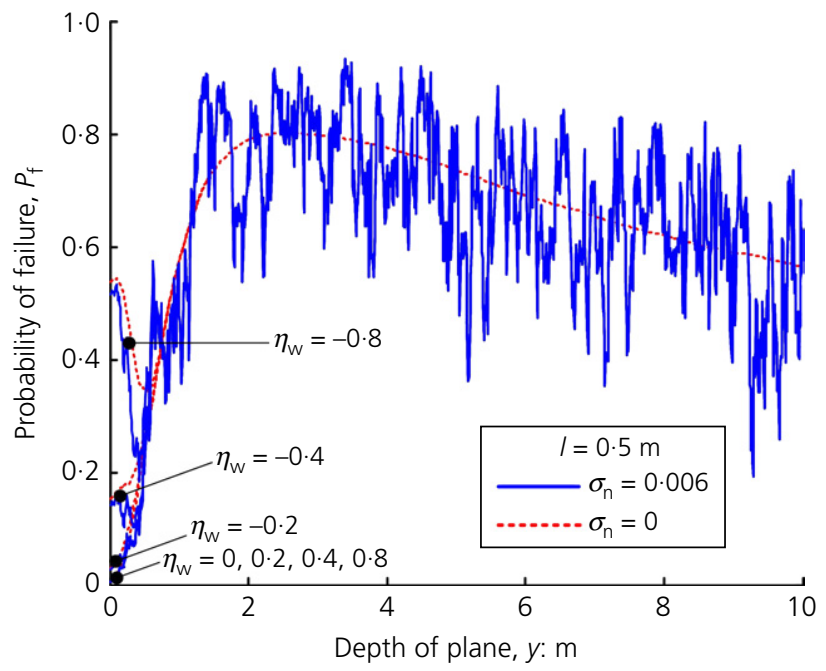

Figure 8. Probabilities of failure for a slope with a shallow groundwater level

groundwater level when $\eta_{\mathrm{w}}=-0 \cdot 2$. The fluctuation of soil porosity will cause the fluctuation of probability of failure along the depth, especially when the failure plane is beneath the groundwater level.

\subsection{Scenario 3: Shallow groundwater level $(I=0.5 \mathrm{~m})$}

Figure 8 shows the probability of failure $P_{\mathrm{f}}(y)$ with respect to the location of the failure plane when the groundwater level is set to be $0.5 \mathrm{~m}$. The normalised infiltration ratio $\left(\eta_{\mathrm{w}}\right)$ is set to be from -0.8 (rainfall) to 0.8 (evaporation). The value of $\sigma_{\mathrm{m}}$ is set to be 0 and 0.006 for monotonic and fluctuating changes of soil porosity along the depth, respectively. As shown in 
Figure 8, although the increase of rainfall intensity will increase the probability of failure above the groundwater level, the probability of failure is governed by the location of the groundwater level. The most unsafe location is always beneath the groundwater level for all the normalised infiltration ratios. Compared with the first and second scenarios, the fluctuation of soil porosity will cause a distinct fluctuation of probability of failure along the depth for this case.

\section{Conclusions}

A methodology has been proposed in this paper for prediction of the failure of an infinite soil slope subject to steady unsaturated flow conditions. An inversely linearly proportional relationship between the friction angle and its porosity was adopted in the analysis. Probabilistic models for soil properties, namely, soil porosity, friction angle, matric suction and saturation, have been developed. The probability of slope failure for different groundwater levels and infiltration (or evaporation) intensities has been investigated thoroughly. It has been found from numerical results that, for a slope with a deep groundwater level, the slope failure is mainly controlled by the rainfall infiltration, whereas, for a shallow groundwater level, the slope failure is governed by the location of the groundwater level. It has also been found that, for a slope with deep groundwater, the most unsafe location is shallow and above the groundwater level; for medium groundwater, the most unsafe location is deep and beneath the groundwater level; and for shallow groundwater, the most unsafe location is always beneath the groundwater level. A merit of the proposed methodology is that the fluctuation of the soil porosity along the depth has been taken into account and modelled as a Gaussian random field. It can be concluded that the proposed methodology can be used for prediction of slope failures under steady unsaturated flow conditions.

\section{Acknowledgement}

Financial support from Australian Research Council under DP140101547, DE130101342 and LP150100413 is gratefully acknowledged.

\section{REFERENCES}

Brooks R and Corey A (1964) Hydraulic Properties of Porous Media. Colorado State University, Fort Collins, CO, USA, Hydrology Paper No. 3.

Carrier W (2003) Goodbye, Hazen; Hello, Kozeny-Carman. Journal of Geotechnical and Geoenvironmental Engineering 129(11): 1054-1056.

Cho SE (2014) Probabilistic stability analysis of rainfall-induced landslides considering spatial variability of permeability. Engineering Geology 171: 11-20.

Cho $S$ and Lee S (2002) Evaluation of surficial stability for homogeneous slopes considering rainfall characteristics. Journal of Geotechnical and Geoenvironmental Engineering 128(9): 756-763.
Cornforth DH (2005) Landslides in Practice: Investigation, Analysis, and Remedial/Preventative Options in Soils. Wiley, Hoboken, NJ, USA.

Escario V and Juca JFT (1989) Strength and deformation of partly saturated soils. Proceedings of the 12th International Conference on Soil Mechanics and Foundation Engineering (ICSMFE), Rio de Janeiro, Brazil, pp. 43-46.

Fenton GA and Griffiths DV (2008) Risk Assessment in Geotechnical Engineering. Wiley, Hoboken, NJ, USA.

Fredlund DG and Xing A (1994) Equations for the soil-water characteristic curve. Canadian Geotechnical Journal 31(3): 521-532.

Fredlund DG, Xing A, Fredlund MD and Barbour SL (1996) Relationship of the unsaturated soil shear strength to the soil-water characteristic curve. Canadian Geotechnical Journal 33(3): 440-448.

Gallipoli D, Wheeler SJ and Karstunen M (2003) Modelling of variation of degree of saturation in a deformable unsaturated soil. Géotechnique 53(1): 105-112.

Gardner W (1956) Mathematics of isothermal water conduction in unsaturated soils. In Highway Research Board Special Report 40, International Symposium on Physico-Chemical Phenomenon in Soils. Highway Research Board, Washington, DC, USA, pp. $78-87$.

Griffiths D and Fenton GA (1993) Seepage beneath water retaining structures founded on spatially random soil. Géotechnique 43(4): 577-587.

Griffiths DV and Smith IM (1991) Numerical Methods for Engineers, 2nd edn. CRC Press, Boca Raton, FL, USA

Griffiths DV, Huang J and Fenton GA (2011) Probabilistic infinite slope analysis. Computers and Geotechnics 38(4): $577-584$.

Iverson RM (2000) Landslide triggering by rain infiltration. Water Resources Research 36(7): 1897-1910.

Li WC, Lee LM, Cai H et al. (2013) Combined roles of saturated permeability and rainfall characteristics on surficial failure of homogeneous soil slope. Engineering Geology 153: 105-113.

Lu N and Godt J (2008) Infinite slope stability under steady unsaturated seepage conditions. Water Resources Research 44(11): W11404.

Lu N and Griffiths D (2004) Profiles of steady-state suction stress in unsaturated soils. Journal of Geotechnical and Geoenvironmental Engineering 130(10): 1063-1076.

Mitchell JK, Houston WN, Scott RF, Carrier WD and Bromwell LG (1972) Mechanical properties of lunar soil: density, porosity, sohesion, and angle of internal friction. In Proceedings of the 3rd Lunar Science Conference (King EA (ed.)). The MIT Press, Cambridge, MA, USA, pp. 3235-3253.

Mualem Y (1976) A new model for predicting the hydraulic conductivity of unsaturated porous media. Water Resources Research 12(3): 513-522. 
Phoon KK and Kulhawy FH (1999) Characterization of geotechnical variability. Canadian Geotechnical Journal 36(4): 612-624.

Ray R, Jacobs J and de Alba P (2010) Impacts of unsaturated zone soil moisture and groundwater table on slope instability. Journal of Geotechnical and Geoenvironmental Engineering 136(10): 1448-1458.

Rowe PW (1969) The relation between the shear strength of sands in triaxial compression, plane strain and direct shear. Géotechnique 19(1): 75-80.

Santoso AM, Phoon KK and Quek ST (2011) Effects of soil spatial variability on rainfall-induced landslides. Computers and Structures 89(11-12): 893-900.

Selby MJ (1993) Hillslope Materials and Processes. Oxford University Press, Oxford, UK.

Sheng D (2011) Review of fundamental principles in modelling unsaturated soil behaviour. Computers and Geotechnics 38(6): 757-776.

Sheng D and Zhou AN (2011) Coupling hydraulic with mechanical models for unsaturated soils. Canadian Geotechnical Journal 48(5): 826-840.

Sheng D, Zhou AN and Fredlund DG (2011) Shear strength criteria for unsaturated soils. Geotechnical and Geological Engineering 29(2): 145-159.

Sun DA, Sheng D, Cui HB and Sloan SW (2007) A densitydependent elastoplastic hydro-mechanical model for unsaturated compacted soils. International Journal for
Numerical and Analytical Methods in Geomechanics 31(11): 1257-1279.

Tarantino A (2009) A water retention model for deformable soils. Géotechnique 59(9): 751-762.

Thu TM, Rahardjo H and Leong EC (2007) Critical state behavior of a compacted silt specimen. Soils and Foundations 47(4): 749-755.

Travis Q, Houston S, Marinho F and Schmeeckle M (2010) Unsaturated infinite slope stability considering surface flux conditions. Journal of Geotechnical and Geoenvironmental Engineering 136(7): 963-974.

van Genuchten MT (1980) A closed-form equation for predicting the hydraulic conductivity of unsaturated soils. Soil Science Society of America Journal 44(5): 892-898.

Vanmarcke EH (1977) Probabilistic modeling of soil profiles. Journal of Geotechnical Engineering, ASCE 103(11): 1227-1246.

Zhang J, Huang HW, Zhang LM, Zhu HH and Shi B (2014) Probabilistic prediction of rainfall-induced slope failure using a mechanics-based model. Engineering Geology 168: $129-140$.

Zhou AN, Sheng D and Carter JP (2012) Modelling the effect of initial density on soil-water characteristic curves. Géotechnique 62(8): 669-680.

Zhu H, Zhang LM, Zhang LL and Zhou CB (2013) Two-dimensional probabilistic infiltration analysis with a spatially varying permeability function. Computers and Geotechnics 48: 249-259.

\section{HOW CAN YOU CONTRIBUTE?}

To discuss this paper, please email up to 500 words to the editor at journals@ice.org.uk. Your contribution will be forwarded to the author(s) for a reply and, if considered appropriate by the editorial board, it will be published as discussion in a future issue of the journal.

Proceedings journals rely entirely on contributions from the civil engineering profession (and allied disciplines). Information about how to submit your paper online is available at www.icevirtuallibrary.com/page/authors, where you will also find detailed author guidelines. 